\title{
L'étalement résidentiel des métropoles intermédiaires de la région Centre Val de Loire
}

The urban sprawl of mid-sized cities in the Centre Val-de-Loire region

Expansión residencial de las ciudades intermedias en la Región Central del Valle del Loira

Jean-Marc Zaninetti et Dominique Andrieu

\section{OpenEdition \\ Journals}

Édition électronique

URL : http://journals.openedition.org/mappemonde/310

DOI : 10.4000/mappemonde.310

ISSN : $1769-7298$

Éditeur

UMR ESPACE

\section{Référence électronique}

Jean-Marc Zaninetti et Dominique Andrieu, « L'étalement résidentiel des métropoles intermédiaires de la région Centre Val de Loire», Mappemonde [En ligne], 124 | 2018, mis en ligne le 10 juillet 2018, consulté le 20 juin 2019. URL : http://journals.openedition.org/mappemonde/310 ; DOI : 10.4000/ mappemonde.310

Ce document a été généré automatiquement le 20 juin 2019.

\section{cc) (†) (ㅇ)}

La revue Mappemonde est mise à disposition selon les termes de la Licence Creative Commons Attribution - Pas d'Utilisation Commerciale - Partage dans les Mêmes Conditions 4.0 International. 


\title{
L'étalement résidentiel des métropoles intermédiaires de la région Centre Val de Loire
}

\author{
The urban sprawl of mid-sized cities in the Centre Val-de-Loire region \\ Expansión residencial de las ciudades intermedias en la Región Central del Valle \\ del Loira
}

Jean-Marc Zaninetti et Dominique Andrieu

\section{Introduction et problématique}

1 L'équipement automobile de tous les ménages français durant le dernier demi-siècle écoulé a métamorphosé la morphologie des villes (Dupuy, 1999). L'espace-temps s'est contracté, les bassins d'emploi urbains se sont dilatés, ce qui a ouvert une abondance de gisements fonciers au développement résidentiel (Halleux, 2011) et provoqué la naissance de la «ville diffuse » (Indovina, 1990) dépourvue de limites clairement définies (Lang, 2002). Ce mouvement d'éparpillement périurbain de l'habitat s'est constamment amplifié depuis son origine (Julien, 2000 ; Baccaïni et Sémécurbe, 2009).

2 Le constat de cette nouvelle forme d'étalement résidentiel autour des villes conduit les géographes français à adopter très vite les néologismes de "périurbanisation»/« rurbanisation» (Bauer et Roux, 1976). La prise en compte institutionnelle de cette nouvelle réalité territoriale amène l'INSEE à définir et à délimiter un nouveau zonage en aires urbaines fonctionnelles en 1994 (Le Jeannic, 1997). Dans celui-ci, les campagnes annexées par l'INSEE au sein des aires urbaines sont dénommées « couronne des pôles urbains ».

3 La soutenabilité environnementale de la ville étalée est controversée (Nessi et al., 2016), et on lui oppose volontiers le contre-modèle de la ville dense, supposée optimiser la consommation d'énergie (Desjardins, 2010). La critique de la ville diffuse repose souvent sur des préjugés négatifs (PUCA, 2014). Le débat porte principalement sur la densité 
urbaine. Désirable pour les uns parce qu'entre autres qualités, elle permet d'offrir des alternatives à la dépendance automobile (Newman et Kenworthy, 1999; Fouchier, 1998 ; Wiel, 2002 ; Pouyanne, 2004), la densité urbaine reste discutée (Charmes, 2010 ; Guignard et Marie, 2017). On observe d'ailleurs un mouvement de balancier sur cette question, et après avoir été jugé « insoutenable » (Berque et al., 2006) ou « incontrôlable » (Djellouli et al., 2010), l'étalement "périurbain» est aujourd'hui requalifié avec une nouvelle appellation jugée plus valorisante, celle de « campagnes urbaines » (Bonnet, 2016).

Parallèlement à ces débats, le législateur s'est alarmé du gaspillage foncier que l'on observe en France et a souhaité préserver le patrimoine de terres agricoles et d'espaces naturels supposés grignotés par l'urbanisation (Charmes, 2013). Adoptée en décembre 2000 et amendée en 2002 et 2003, la loi dite de «Solidarité et Renouvellement urbain » (SRU) marque un remaniement en profondeur des règles d'aménagement et d'urbanisme qui prévalaient en France depuis la Loi d'orientation foncière (LOF) de 1967 (dite «Loi Pisani ») qui avait introduit le principe de l'urbanisme de zonage dans le droit français (Goze, 2002). La spécialisation fonctionnelle des espaces urbanisés avait d'ailleurs été jugée créatrice de dépendance automobile (Sainteny, 2008). Le nouveau cadre réglementaire permet un retour à une mixité fonctionnelle limitée dans les nouveaux aménagements (Renard, 2003). Ses dispositions en faveur de la densification urbaine pour une gestion économe des ressources foncières ont été renforcées par la suite avec la loi « portant engagement national pour l'environnement » (loi dite « Grenelle II », 2010) et la « loi pour l'accès au logement et un urbanisme rénové » (ALUR, 2014) (Touati et Crozy, 2015).

Dans le contexte de la décentralisation, la concurrence entre les collectivités avait été considérée comme un facteur de surconsommation foncière. On a parlé de «ville fragmentée » (Zaninetti et Maret, 2008) ou de «ville émiettée » (Charmes, 2011). C'est pourquoi la loi SRU a d'abord voulu renforcer la gouvernance territoriale afin de restreindre l'offre foncière potentiellement urbanisable (Halleux, 2011). Cette loi entend remédier à la fragmentation communale en établissant une hiérarchie de documents d'urbanisme opposables (Goze, 2002). Elle a également cherché à promouvoir la reconstruction de la ville sur elle-même, qualifiée de "renouvellement urbain» (Bonneville, 2004). Dans le détail, la loi SRU a également pris des dispositions techniques levant les restrictions réglementaires qui s'opposaient à la densification urbaine (Goze, 2002). Toutefois, le changement réglementaire tarde à produire des effets mesurables sur la densité urbaine en général et résidentielle en particulier (Touati et Crozy, 2015).

Pour autant, il ne faut pas négliger l'inscription de la croissance urbaine dans la durée. D'après la dernière livraison des enquêtes annuelles de recensement, par exemple, moins de $7 \%$ du parc de logement achevé avant le $1^{\mathrm{er}}$ janvier 2012 en région Centre-Val de Loire a été construit dans les 5 années précédentes. Si d'autres régions en expansion ont des parcs de logement en croissance plus rapide (plus de $9 \%$ de logements récents dans les régions du Grand Ouest, Bretagne et Pays de la Loire), la région Centre-Val de Loire n'en est pas moins située dans la moyenne nationale. Au terme d'un demi-siècle d'étalement, la ville éparpillée dans ses campagnes urbaines environnantes héberge un français sur quatre, sur $40 \%$ du territoire (Floch et Levy, 2011). C'est un héritage avec lequel il convient de composer aujourd'hui (Nessi et al., 2016). Si l'injonction à la densification résidentielle est opérationnelle depuis le tournant du siècle, elle doit pouvoir se mesurer graduellement dans les données disponibles sur le terrain. C'est pourquoi nous voulons confronter le débat théorique à l'épreuve du terrain à partir de l'étude de cas empirique 
des deux métropoles de la Loire moyenne, Orléans et Tours. Notre modeste ambition est de rendre compte des formes spatiales et des rythmes de l'étalement urbain autour de deux "métropoles $»^{1}$ de la région Centre-Val de Loire. Ces deux villes ressortaient en position moyenne dans l'étude de l'intensité de la périurbanisation autour des villes françaises publiée en 1999 (Wiel, 1999). Cet article tente de mesurer l'évolution des modalités d'urbanisation de deux aires urbaines moyennes en lien avec l'évolution des politiques publiques nationales et locales, entre injonctions, adaptations et résistances. Notre étude de cas s'inscrit dans le cadre conceptuel qui a été discuté précédemment. Sans prétendre élaborer une théorie nouvelle, elle sert à relativiser les modèles et les questions théoriques dans le contexte régional en vue d'orienter la suite du programme de recherche dans lequel elle s'inscrit.

\section{Méthodologie de l'étude}

$7 \quad$ Notre étude repose sur l'exploitation des fichiers fonciers MAJIC².

\section{Les fichiers fonciers MAJIC}

8 Cette source administrative a été constituée à des fins fiscales par la direction générale des impôts, mais elle est utilisée pour les études sur l'étalement urbain au sein des administrations concernées (DREAL Franche Comté, 2013). L'intérêt pour cette source en géographie urbaine provient de la possibilité qu'elle offre de mobiliser une information à grande échelle, indispensable pour prendre la mesure des contraintes naturelles, que ce soit en montagne (Fablet, 2013) ou sur le littoral par exemple (Le Berre et al., 2016), en particulier dans l'étude des enjeux exposés aux risques naturels (Chauveau et al., 2012). L'utilisation de MAJIC qui est la nôtre pour mettre en évidence la diffusion de la tache urbaine a au moins un précédent, justement sur le cas de l'agglomération de Tours (Polombo et Beauvais, 2014). La date de construction et la superficie des locaux d'activité sont malheureusement mal renseignées dans la source, ce qui restreint son domaine d'application à l'étude de l'étalement résidentiel seulement. Quelques variables d'identification tirées du fichier des propriétés bâties ont permis d'établir une typologie simplifiée du logement, collectif ou individuel, et de connaître les dates d'achèvement de ces logements ainsi que leur surface habitable. Le parc de logement a été géocodé sur la BD parcellaire de l'IGN. Toutefois, l'analyse des données à l'échelle des aires urbaines d'Orléans et de Tours ne permet pas d'exploiter directement la localisation à la parcelle, celle-ci contient d'ailleurs quelques défauts de précision, identifiés dans une phase exploratoire de contrôles de qualité. Les logements sont donc agrégés sur un carroyage, conformément aux recommandations méthodologiques du ministère responsable de l'environnement, de l'aménagement et du logement en matière de transports et d'urbanisme (IETI Consultants, 2008). Le découpage choisi est le carroyage de diffusion de l'INSEE. Il dessine des carreaux de 4 hectares, soit 200 mètres de côté. Il a été réalisé à l'INSEE dans le cadre d'une cartographie homogène de la densité de population en Europe supervisée par EUROSTAT, l'organisme statistique des communautés européennes. Notre objectif est d'étudier les rythmes de construction du parc existant au $1^{\text {er }}$ janvier 2013, ainsi que la répartition de ce parc entre maisons et appartements.

9 Les fichiers MAJIC ne sont pas des fichiers historiques. Les logements démolis ne sont pas conservés dans la base de données, ce qui signifie que nous observons l'ancienneté du 
parc existant à la date d'exploitation de la base et non l'ensemble de l'historique du parc. La source est donc mieux adaptée à l'étude de l'étalement urbain qu'à celle de la densification par le renouvellement urbain.

\section{Périmètre de l'étude et indicateurs retenus}

10 La difficulté de fixer une limite entre l'espace urbain et rural, alors même qu'ils se mêlent l'un dans l'autre, rend arbitraire tout essai de délimitation d'un périmètre d'étude. Toutefois, pour comprendre l'évolution des espaces urbanisés, il est nécessaire que les aires urbaines INSEE composent le cadre territorial de notre étude de cas. L'étalement résidentiel des deux villes est étudié dans le périmètre constant des aires urbaines dans leurs limites de 2010. Il semble pertinent de maintenir un cadre spatial fixe sur l'ensemble de la période considérée (Julien, 2007), qui s'étend entre 1945 et 2013, pour mettre l'accent sur la construction de logement, sans que la question épineuse de l'expansion des périmètres ne complique la mesure (Le Jeannic, 1997 ; Wiel, 1999 ; Bessy-Pietri, 2000 ; Julien, 2000). Nous adoptons donc une définition fonctionnelle de l'urbain caractérisé par l'espace des déplacements domicile - travail. Les aires urbaines de l'INSEE nous procurent un cadre géographique reconnu, même s'il ne faut pas oublier les imperfections de ce zonage (Drevelle, 2012 ; Lévy, 2013 ; Zaninetti, 2017). Toutes les statistiques qui suivent sont calculées à périmètre constant.

11 Le rapprochement des fichiers MAJIC du ministère du Logement et de la BD cadastrale DGI-IGN permet de calculer un indicateur de densité résidentielle finement localisé. Il s'agit du rapport entre la surface habitable des logements $\left(e n \mathrm{~m}^{2}\right.$ ) et la superficie parcellaire des terrains sur lesquels ils sont bâtis. Ce « Coefficient d'Occupation des Sols » (COS) résidentiel observé ne doit pas être confondu avec le CoS réglementaire, qui a longtemps été le plafond de densité fixé par les documents d'urbanisme avant d'être supprimé par la loi ALUR (Touati et Crozy, 2015). En pratique, le COS résidentiel observé est inférieur aux anciens COS réglementaires, en dehors des noyaux urbains les plus denses. Par ailleurs, on peut aussi se contenter d'un dénombrement des logements selon le type simplifié séparant maisons et appartements dans la source ou de manière agrégée sur le carroyage. Ces indicateurs sont analysés de manière longitudinale, en premier lieu, pour comprendre les rythmes de la croissance urbaine autour d'Orléans et de Tours après la Seconde Guerre mondiale, et de manière spatio-temporelle, en second lieu, pour décrire la géographie de ces extensions.

\section{L'expansion résidentielle d'Orléans et de Tours}

Tableau 1. Comparaison des aires urbaines d'Orléans et de Tours (délimitation 2010)

\begin{tabular}{|l|l|l|}
\hline Indicateur au 1er janvier 2013 & $\begin{array}{l}\text { Aire urbaine } \\
\text { d'Orléans }\end{array}$ & $\begin{array}{l}\text { Aire urbaine de } \\
\text { Tours }\end{array}$ \\
\hline Nombre de communes & 138 & 144 \\
\hline Superficie & $3100 \mathrm{~km}^{2}$ & $3190 \mathrm{~km}^{2}$ \\
\hline Part du foncier bâti (\% de la superficie cadastrale) & $6,7 \%$ & $10,4 \%$ \\
\hline
\end{tabular}




\begin{tabular}{|l|l|l|}
\hline $\begin{array}{l}\text { CoS résidentiel observé (MAJIC, } \mathrm{m}^{2} \text { habitable } / \mathrm{m}^{2} \text { de } \\
\text { terrain) }\end{array}$ & 0,11 & 0,08 \\
\hline Nombre de logements au $1^{\mathrm{er}}$ janvier 2013 (MAJIC) & 216810 & 254225 \\
\hline
\end{tabular}

Sources : INSEE, recensement, IGN, Geofla, BDTopo, ministère du Logement, MAJIC

tableau 1). Par contre, l'aire urbaine de Tours est plus peuplée que celle d'Orléans. Sur ce territoire de référence, l'urbanisation est sensiblement plus étalée autour de Tours que d'Orléans. Dans les deux cas, la préférence pour l'habitat individuel est très marquée. Selon l'INSEE, tandis que 55,8\% du parc de logement est constitué de maisons au recensement 2013 en France métropolitaine, le même indicateur se situe 3 points audessus de la moyenne dans les aires urbaines d'Orléans et de Tours (moyenne de 58,9\% selon le recensement).

13 La vallée de la Loire moyenne appartient à l'aire culturelle de la France du Nord-Ouest qui s'étend à l'ouest d'une ligne imaginaire reliant Lille à Bordeaux. Dans cette France de l'Ouest, la tradition de l'habitat individuel était solidement ancrée avant le commencement de la phase d'étalement urbain dans les années 1960 (Wiel, 1999). Une géographie des marchés fonciers agricoles montre que la vallée de la Loire moyenne s'inscrit dans le même modèle que le Grand Ouest, à l'exception du nord-ouest de l'aire urbaine d'Orléans, dont la structure foncière Beauceronne se rattache au modèle du Bassin parisien, plus «résistant " à la pression foncière résidentielle périurbaine (Levesque et al., 2011).

\section{Les rythmes de l'expansion résidentielle}

Les fichiers MAJIC ne conservent pas l'historique des logements qui ont été démolis, il est donc aventureux de remonter à une date antérieure à la fin de la Seconde Guerre mondiale. On observe que $29 \%$ des « logements » enregistrés dans les fichiers fonciers au $1^{\mathrm{er}}$ janvier 2013 ont été construits avant le $1^{\mathrm{er}}$ janvier 1946 dans l'aire urbaine de Tours. À ce stock initial se sont ajoutés 2691 logements nouveaux construits chaque année en moyenne de 1946 à 2012 inclus. Toutefois, le rythme de construction neuve a beaucoup fluctué d'année en année (figure 1). 
Figure 1. Aire urbaine de Tours, graphique de la construction annuelle de logement 1946-2012

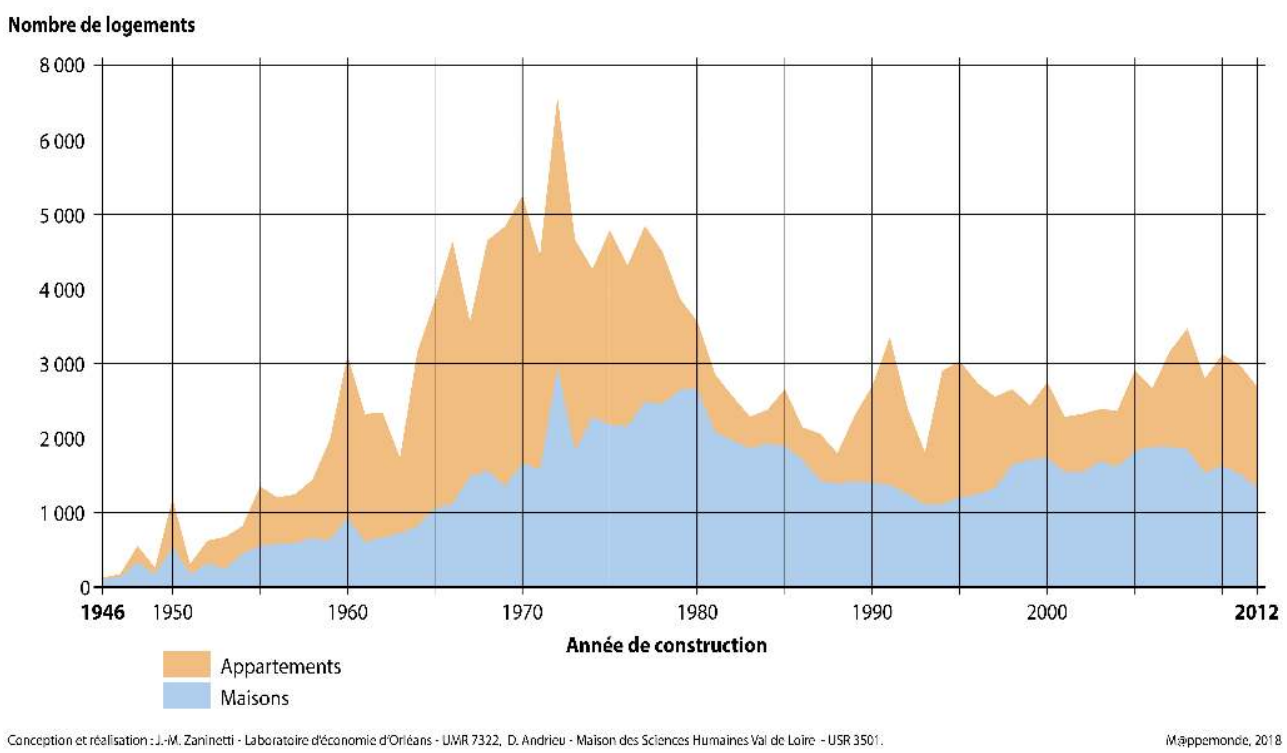

Conception et réalisation : J.-M. Zaninetti - Laboratoire d'écononomie d'Orléans - UMR 7322, D. Andrieu - Maison des Sciences Humaines Val de Loire - USR 3501

15 Les constructions neuves ont été peu nombreuses avant 1960. Une période de construction intensive dure de 1962 à 1982, en 20 ans le parc de logement de l'agglomération tourangelle double d'importance. Près de 4200 logements neufs sont livrés en moyenne chaque année durant cette période à Tours et dans les communes environnantes, soit $33 \%$ du parc existant en 2013. Beaucoup d'appartements sont construits durant cette période de l'urbanisme des grands ensembles triomphant (Roux, 2006). Toutefois, la construction de maisons individuelles monte aussi en puissance inexorablement et représente la quasi-totalité des constructions neuves vers la fin de cette phase de croissance. Après avoir fait de l'urbanisme des grands ensembles une véritable doctrine d'état lorsque, au début des années 1960, la priorité était de résorber la pénurie de logements de l'après-guerre et de loger les rapatriés d'Algérie en multipliant les «Zones d'Urbanisation Prioritaires» (ZUP), les priorités de l'état se déplacent et la période des ZUP prend fin avec la circulaire Guichard de 1973. Au contraire, le Rapport Mayoux fait implicitement l'éloge de l'habitat pavillonnaire (Mayoux, 1979). Des politiques d'aide à la personne sont substituées à l'aide à la pierre (1977). L'accession à la propriété est encouragée par une politique des prêts conventionnés. Ces nouvelles politiques se traduisent par la montée en puissance de la construction de maisons neuves (Taffin, 1987).

Après cette phase d'urbanisation intensive, la production de logements neufs s'effondre durant les années 1980 pour atteindre son minimum en 1988. Le malthusianisme foncier des communes au moment de la décentralisation est une explication couramment avancée (Roux, 2006). Toutefois, on observe que la baisse résulte avant tout de la quasidisparition de la construction d'appartements neufs, alors que la production de maisons individuelles reste soutenue, bien que ralentie par rapport aux années 1970. Hostile aux propriétaires bailleurs, la loi Quillot de 1982 a paralysé la construction de logement locatif privé neuf. La loi Méhaignerie de 1986 abroge la loi Quillot et met en place des politiques de soutien à l'investissement locatif privé qui relancent - à grand frais - 
l'activité dans le secteur de la construction (Gilli, 2006). Les premières livraisons arrivent vers 1989. La construction s'est ralentie à une moyenne de moins de 2500 logements neufs par an en Touraine durant les deux dernières décennies du $20^{\mathrm{e}}$ siècle. Tandis que les appartements représentaient $57 \%$ des livraisons de logements neufs en Touraine entre 1962 et 1982, ils n'en représentaient plus que 40 \% entre 1982 et 1999.

En dépit du changement réglementaire majeur depuis la loi SRU, la construction de logement est restée stable depuis le début du XXI ${ }^{e}$ siècle. Un peu plus de 2700 logements neufs ont été construits en moyenne depuis 1999, dont $39 \%$ d'appartements et $61 \%$ de maisons individuelles. Au fur et à mesure que l'agglomération de Tours a pris de l'importance, la prédominance de l'habitat individuel, historiquement très marquée, a cédé la place à un parc de logement plus diversifié. $72 \%$ des logements existants au $1^{\text {er }}$ janvier 2013 construits avant 1946 étaient des maisons, tandis que la part des maisons n'est plus que de $57 \%$ du parc en 2013.

Dans l'aire urbaine d'Orléans, on observe que $27 \%$ des logements enregistrés dans les fichiers fonciers au $1^{\mathrm{er}}$ janvier 2013 ont été construits avant le $1^{\mathrm{er}}$ janvier 1946. À ce stock initial se sont ajoutés 2358 logements nouveaux construits chaque année, en moyenne, de 1946 à 2012 inclus. La courbe de construction de l'aire urbaine d'Orléans est très similaire à celle de Tours, de sorte qu'il est inutile de revenir sur les facteurs généraux expliquant la même périodisation (figure 2).

Figure 2. Aire urbaine d'Orléans, graphique de la construction annuelle de logement 1946-2012

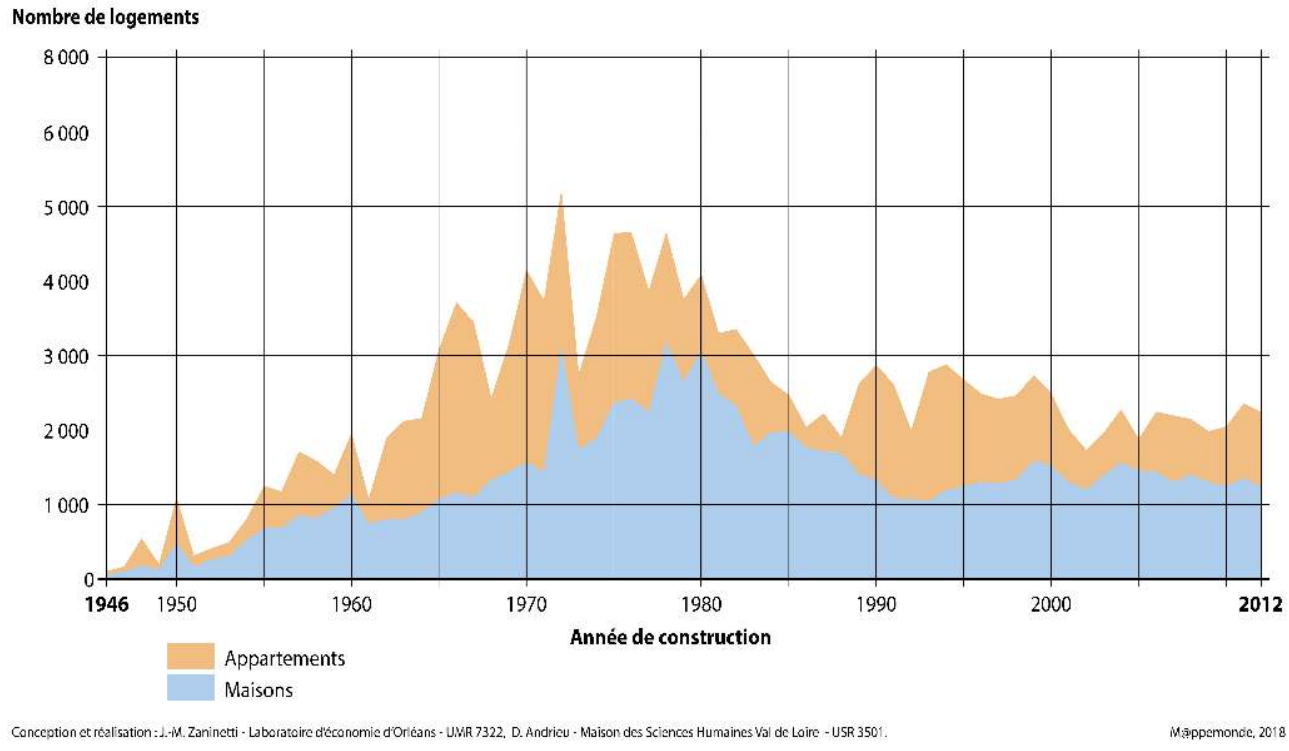

Conception et réalisation : J.-M. Zaninetti - Laboratoire d'écononomie d'Orléans - UMR 7322, D. Andrieu - Maison des Sciences Humaines Val de Loire - USR 3501

Comme l'agglomération orléanaise est plus petite que celle de Tours, le rythme de construction est aussi un peu plus lent. Le rythme annuel moyen de construction culmine avec 3500 logements neufs livrés chaque année dans les décennies 1960 et 1970, dont $48 \%$ d'appartements en immeubles collectifs. Comme à Tours, $33 \%$ du parc de logement existant au $1^{\text {er }}$ janvier 2013 a ainsi été construit entre 1962 et 1981 . Cette période d'urbanisation rapide est suivie de la même crise du logement dans les années 1980, puis de la même reprise à partir des années 1990. Le rythme de construction neuve tombe en moyenne à 2500 logements par an dans les deux dernières décennies du XX $\mathrm{XX}^{\mathrm{e}}$ siècle, dont 
$41 \%$ d'appartements, avant de baisser encore à moins de 2150 unités par an, dont $36 \%$ d'appartements, depuis le tournant du siècle. La divergence observable entre Tours et Orléans depuis le début du XXI siècle s'explique peut-être par la perte d'attractivité de l'aire urbaine d'Orléans observée après le recensement de 1999, alors que la Touraine a continué à se développer au rythme moyen national. Tours dispose d'une université qui accueille deux fois plus d'étudiants que celle d'Orléans, avec en particulier une faculté de médecine qui est absente à Orléans, ce qui influe de manière significative sur son solde migratoire. En conséquence, la part du logement collectif est moins développée à Orléans qu'à Tours. En dépit du développement urbain survenu dans la seconde moitié du $\mathrm{XX}^{\mathrm{e}}$ siècle, les maisons représentent encore $59 \%$ du parc existant en 2013 dans l'Orléanais, contre $67 \%$ en 1946.

\section{La densité urbaine}

20 Tandis que les rythmes et la répartition de la construction de logement neuf entre maisons et appartements sont très similaires dans les deux aires urbaines de 1946 à 2012, l'évolution des densités résidentielles présente aussi de grandes similitudes.

21 Le COS résidentiel constaté reflète l'équilibre général du marché du logement. Son comportement est complexe. Il fluctue soit sous l'influence de la réglementation, soit sous celle du marché. Si les collectivités augmentent les COS réglementaires, ils favorisent l'augmentation du COS résidentiel effectif, mais cet effort peut être contrarié sur un marché dominé par la construction de logements individuels par des gains de pouvoir d'achat qui permettent, en l'absence de contrainte foncière, d'agrandir la taille des parcelles de terrain à bâtir. Dans les communes d'urbanisation diffuse, l'élaboration d'un Plan d'occupation des sols est souvent associée à une politique de peuplement (Duvernoy, 2002). L'action sur les COS réglementaires a une influence sur les valeurs foncières (Comby, 2004). Le maintien de COS réglementaires planchers crée un filtre économique qui évince le logement locatif et sélectionne les acquéreurs qui peuvent acheter des parcelles plus grandes (Lambert, 2013). Mais une montée des prix fait pression sur les collectivités locales pour qu'elles relèvent les $\cos$ si elles veulent attirer des constructions nouvelles. Sur la longue durée, un suivi des prix est impossible, et devrait aussi tenir compte de l'évolution des revenus pour être apprécié en termes relatifs. Par contre, l'observation des COS résidentiels traduit le " pouvoir d'achat » foncier relatif de la ville sur les terrains environnants. Soit la ville se construit en déversant ses constructions nouvelles sur des terrains qui n'étaient pas urbanisés précédemment, auquel cas les COS résidentiels observés restent stables ou diminuent si le "pouvoir d'achat » relatif de la demande résidentielle augmente, soit la ville doit se densifier avec une ressource foncière contrainte et les $\operatorname{CoS}$ résidentiels augmentent.

La comparaison entre les deux aires urbaines ligériennes indique que, sur la longue durée, la densité urbaine reste faible (tableau 2). Le CoS résidentiel observé passe de 0,05 en 1945 à 0,08 en 2013 en Touraine, tandis que la progression est très modeste dans l'Orléanais, 0,11 en 2013 contre 0,09 en 1945. Pour l'essentiel, les deux agglomérations se sont développées en s'étalant, c'est-à-dire en construisant de nouveaux logements principalement en périphérie. Toutefois, dans le détail, on observe des différences entre les deux aires urbaines. 
Tableau 2. Comparaison statistique Orléans/Tours selon l'ancienneté du parc de logement

\begin{tabular}{|c|c|c|c|c|c|c|}
\hline \multirow[b]{2}{*}{$\begin{array}{l}\text { Logement } \\
\text { selon les } \\
\text { dates de } \\
\text { construction }\end{array}$} & \multicolumn{3}{|c|}{ Aire urbaine de Tours } & \multicolumn{3}{|c|}{ Aire urbaine d'Orléans } \\
\hline & $\begin{array}{l}\text { Ville de } \\
\text { Tours } \\
\text { (délimitation } \\
2013 \text { ) }\end{array}$ & $\begin{array}{l}39 \text { communes } \\
\text { périphériques } \\
\text { SCOT de } \\
\text { Tours }\end{array}$ & $\begin{array}{l}\text { Périphérie } \\
\text { de l'aire } \\
\text { urbaine }\end{array}$ & $\begin{array}{l}\text { Ville } \\
\text { d'Orléans } \\
\text { (délimitation } \\
\text { 2013) }\end{array}$ & $\begin{array}{l}21 \text { communes } \\
\text { périphériques } \\
\text { SCOT } \\
\text { d'Orléans }\end{array}$ & $\begin{array}{l}\text { Périphérie } \\
\text { de l'aire } \\
\text { urbaine }\end{array}$ \\
\hline $\begin{array}{l}\text { Superficie } \\
\text { cadastrale } \\
\text { (\%, total aire } \\
\text { urbaine } 100 \% \\
\text { ) }\end{array}$ & $3 \%$ & $24 \%$ & $73 \%$ & $8 \%$ & $14 \%$ & $78 \%$ \\
\hline $\begin{array}{l}\text { Parc de } \\
\text { logement } \\
\text { construit } \\
\text { avant } 1946 \\
\text { (\%, total aire } \\
\text { urbaine 100\% } \\
\text { ) }\end{array}$ & $\begin{array}{l}24289 \\
(33 \%)\end{array}$ & $\begin{array}{l}20222 \\
(27 \%)\end{array}$ & $\begin{array}{l}29411 \\
(40 \%)\end{array}$ & $\begin{array}{l}19770 \\
(34 \%)\end{array}$ & $\begin{array}{l}9888 \\
(17 \%)\end{array}$ & $\begin{array}{l}29171 \\
(50 \%)\end{array}$ \\
\hline $\begin{array}{l}\text { COS } \\
\text { résidentiel } \\
\text { observé } \\
\text { avant } 1946\end{array}$ & 0,38 & 0,06 & 0,03 & 0,31 & 0,07 & 0,12 \\
\hline $\begin{array}{l}\text { Superficie } \\
\text { moyenne } \\
\text { logement } \\
\text { ancien (ante } \\
1946 \text { ) }\end{array}$ & $64 \mathrm{~m}^{2}$ & $92 \mathrm{~m}^{2}$ & $94 \mathrm{~m}^{2}$ & $67 \mathrm{~m}^{2}$ & $94 \mathrm{~m}^{2}$ & $102 \mathrm{~m}^{2}$ \\
\hline $\begin{array}{l}\text { Part des } \\
\text { maisons } \\
\text { (ante 1946) }\end{array}$ & $37 \%$ & $86 \%$ & $92 \%$ & $31 \%$ & $77 \%$ & $88 \%$ \\
\hline $\begin{array}{l}\text { Parc de } \\
\text { logement } \\
\text { construit de } \\
1946 \text { à } 2012 \\
\text { (\%, total aire } \\
\text { urbaine 100\% } \\
\text { ) }\end{array}$ & $\begin{array}{l}60037 \\
(33 \%)\end{array}$ & $\begin{array}{l}87309 \\
(48 \%)\end{array}$ & $\begin{array}{l}32957 \\
(18 \%)\end{array}$ & $\begin{array}{l}46270 \\
(29 \%)\end{array}$ & \begin{tabular}{|l|}
64587 \\
$(41 \%)$
\end{tabular} & $\begin{array}{l}47124 \\
(30 \%)\end{array}$ \\
\hline
\end{tabular}




\begin{tabular}{|l|l|l|l|l|l|l|}
\hline $\begin{array}{l}\text { Cos } \\
\text { résidentiel } \\
\text { observé } \\
\text { moyen pour } \\
\text { les } \\
\text { constructions } \\
\text { nouvelles } \\
\text { (post 1945) }\end{array}$ & 0,47 & 0,11 & 0,06 & 0,41 & 0,17 & 0,07 \\
\hline $\begin{array}{l}\text { Superficie } \\
\text { moyenne } \\
\text { logement } \\
\text { nouveau } \\
\text { (post 1945) }\end{array}$ & $61 \mathrm{~m}^{2}$ & $88 \mathrm{~m}^{2}$ & $93 \mathrm{~m}^{2}$ & $66 \mathrm{~m}^{2}$ & $87 \mathrm{~m}^{2}$ & $97 \mathrm{~m}^{2}$ \\
\hline $\begin{array}{l}\text { Part des } \\
\text { maisons dans } \\
\text { les } \\
\text { constructions } \\
\text { nouvelles } \\
\text { (post 1945) }\end{array}$ & $12 \%$ & $63 \%$ & $86 \%$ & $17 \%$ & $63 \%$ & $86 \%$ \\
\hline
\end{tabular}

Source : MAJIC

Le fichier MAJIC dénombre un peu plus de 180000 logements construits de 1946 à 2012 dans le périmètre de l'aire urbaine de Tours (figure 3) contre un peu moins de 158000 logements construits sur la même période dans l'aire urbaine d'Orléans (figure 4). Les deux métropoles ligériennes ont en commun que les villes-centres sont relativement peu étendues et ne regroupent qu'un tiers du parc de logement de l'aire urbaine. Par contre, la ville de Tours est sensiblement plus dense que celle d'Orléans. Cette différence était déjà observable en 1945, mais l'écart s'est creusé depuis, car Tours a eu deux maires « inamovibles » qui ont fortement marqué l'image de leur ville, Jean Royer de 1959 à 1995 (Lussault, 1993) et son successeur Jean Germain, maire de 1995 à 2014. Le premier, en particulier, est le prototype du «maire bâtisseur » (Lussault, 1993 ; Dumont, 2005). Il s'est obstiné à faire croître sa ville par tous les moyens, mobilisant tous les terrains disponibles pour construire, surtout durant la période des grands ensembles. Son successeur a conduit une politique différente, basée sur la transformation du tissu urbain et en privilégiant l'intercommunalité au sein de la communauté d'agglomération, une stratégie coopérative en partie contrainte par la raréfaction des disponibilités foncières sur le territoire communal. Tandis que la ville de Tours a cherché à développer une ville dense, la plupart des communes périphériques ont résisté à cette politique en privilégiant l'habitat pavillonnaire, avec quelques exceptions telles que Joué les Tours et Saint-Pierre des Corps qui ont accueilli des grands ensembles pendant la période de «haute croissance » des années 1960 et 1970 . Au final, l'aire urbaine tourangelle oppose un centre urbain dense à une périphérie très diffuse.

La moindre densité résidentielle de la ville d'Orléans traduit aussi une plus faible continuité des politiques municipales. Orléans a connu des alternances politiques qui ont entravé la continuité des politiques de développement. Jean-Pierre Sueur, battu aux élections municipales après avoir dirigé la ville de 1989 à 2001, avait conduit une politique 
de densification urbaine dans la ville centre dont l'effet est perceptible sur la courbe de la figure 4, mais qui a été suivie d'un coup d'arrêt à partir de l'alternance au profit de la droite municipale, plus malthusienne dans ses choix d'urbanisme (Dumont, 2005). Il s'est ainsi construit une proportion plus importante de maisons en ville à Orléans, et les logements y sont plus grands qu'à Tours. Le contraste est moins fort entre la ville-centre et les 21 communes de la périphérie de l'agglomération d'Orléans qu'entre Tours et les autres communes de son périmètre SCOT.

La gouvernance territoriale des deux aires urbaines est une autre différence importante. Même si leurs villescentres et leurs communautés d'agglomération sont à peu près comparables, l'aire urbaine de Tours bénéficie aujourd'hui d'une gouvernance territoriale plus centralisée que celle d'Orléans. Avec le regroupement de 4 établissements publics de coopération intercommunale (EPCI) dans un périmètre SCOT étendu à 40 communes, Tours s'est dotée d'un outil de gouvernance qui lui donne un droit de regard sur $72 \%$ des constructions neuves de l'aire urbaine depuis le tournant du siècle. Orléans, au contraire, n'a pas étendu son SCOT au-delà des limites de son agglomération (22 communes), qui ne regroupe que $61 \%$ des constructions de logement neuf récentes.

Si les structures géopolitiques sont assez différentes, la chronologie de l'étalement urbain est de nouveau très semblable d'une aire urbaine à l'autre (figures 3 et 4 ).

Figure 3. Aire urbaine de Tours, graphique de la densité urbaine de la construction de logement 1946-2012

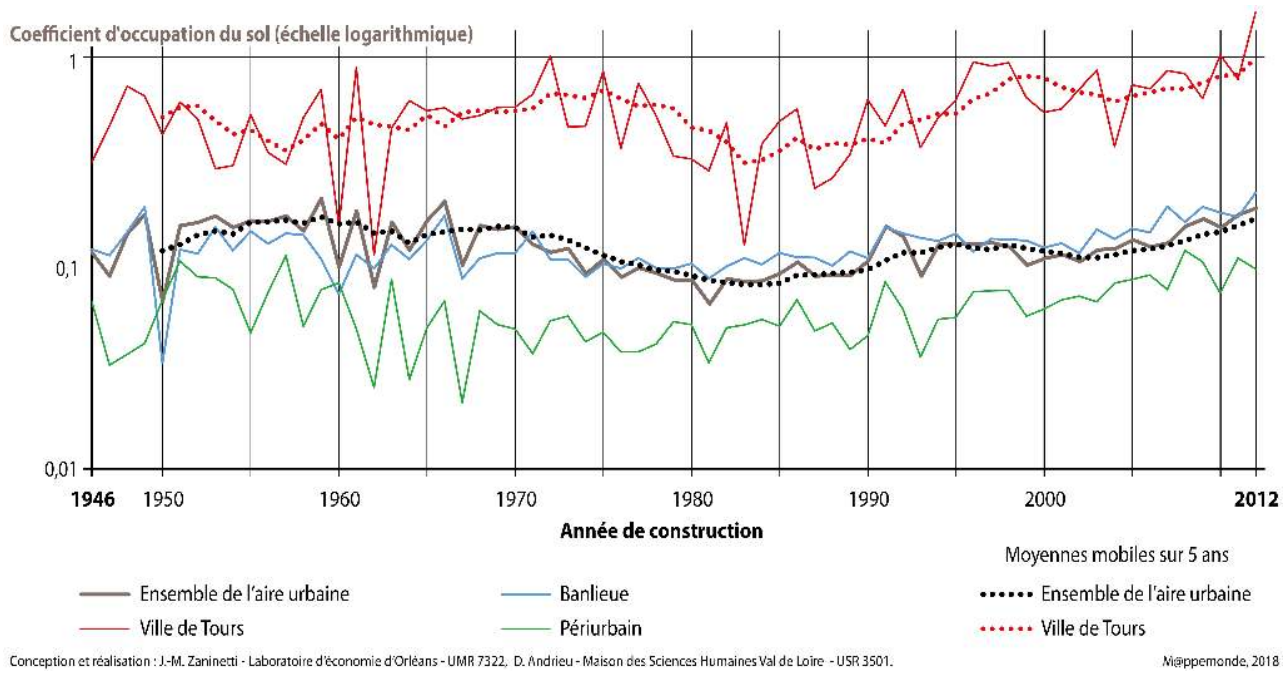

Conception et réalisation : J.-M. Zaninetti - Laboratoire d'écononomie d'Orléans - UMR 7322, D. Andrieu - Maison des Sciences Humaines Val de Loire - USR 3501

D'année en année, le COS résidentiel observé des constructions nouvelles est assez stable dans le périmètre de l'aire urbaine de Tours (figure 3). On observe même une tendance à la baisse de la densité résidentielle pour les constructions neuves de 1946 à 1982 en Touraine, ce qui traduit une urbanisation extensive, prise sur des terrains non bâtis en périphérie avec des parcelles de plus en plus grandes. Toutefois, cette tendance s'est ensuite inversée peu à peu à partir de 1982 pour aboutir à une production de logement plus dense au début du XXI ${ }^{e}$ siècle. Dans le détail, la ville-centre de Tours, qui regroupe $33 \%$ du parc de logement de l'aire urbaine sur son territoire de moins de $35 \mathrm{~km}^{2}$, se distingue de sa périphérie urbaine et périurbaine. En 2013, chaque logement « occupe » en moyenne $141 \mathrm{~m}^{2}$ de terrain à Tours, alors qu'il occupe $1822 \mathrm{~m}^{2}$ de terrain en moyenne 
dans les 39 autres communes du périmètre SCOT (qualifiées de "banlieue» sur ce graphique) qui recouvre le cœur de l'aire urbaine. Le cos observé de la ville de Tours est donc de 0,44 en 2013 contre 0,38 en 1946 dans les limites territoriales de 2013. Inversement, le COS observé des communes périphériques du SCOT est minimal avec 0,05 $\mathrm{m}^{2}$ habitable par $\mathrm{m}^{2}$ de terrain, une densité résidentielle plus faible en 2013 qu'en 1946.

Figure 4. Aire urbaine d'Orléans, graphique de la densité urbaine de la construction de logement 1946-2012

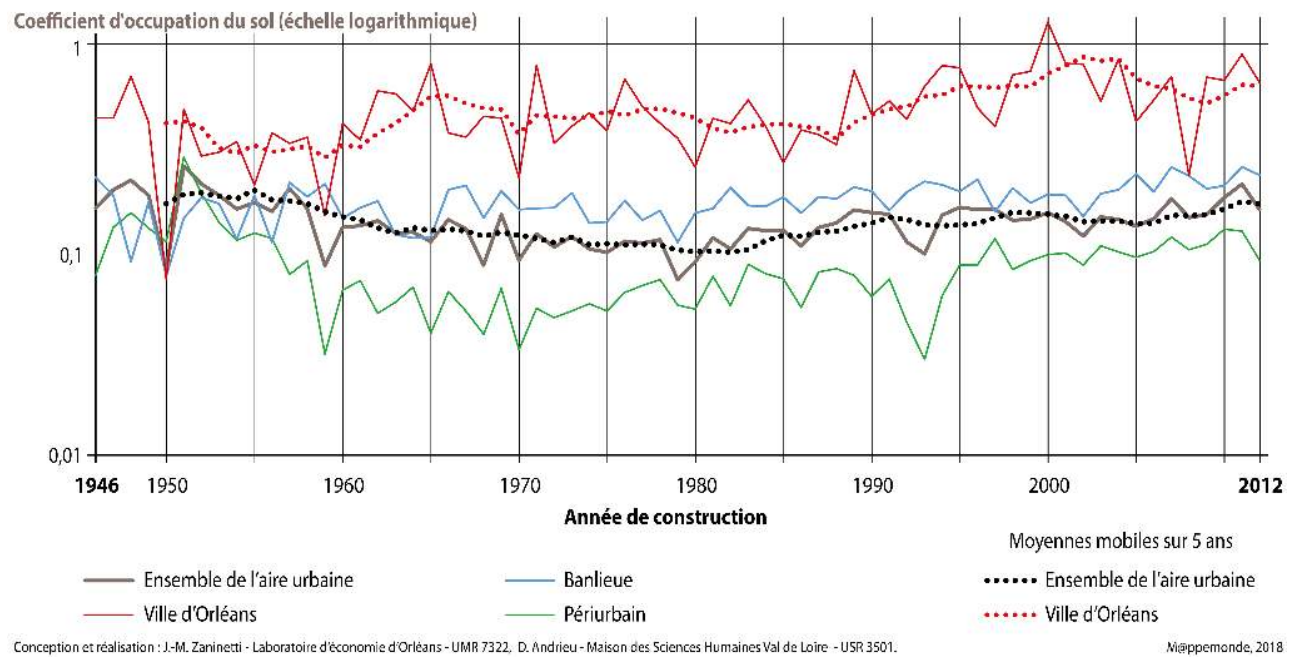

Conception et réalisation : J.-M. Zaninetti - Laboratoire d'écononomie d'Orléans - UMR 7322, D. Andrieu - Maison des Sciences Humaines Val de Loire - USR 3501

Sur cette même période, le CoS résidentiel observé des constructions nouvelles est stable dans le périmètre de l'aire urbaine d'Orléans (figure 4). On observe aussi une tendance à la baisse de la densité résidentielle pour les constructions neuves de 1946 à 1982 dans l'Orléanais. Cette tendance à l'étalement urbain s'est ensuite inversée très modestement à partir de 1982 pour aboutir à une production de logement un peu plus dense au début du $\mathrm{XXI}^{e}$ siècle. Dans le détail, la ville-centre d'Orléans, qui regroupe $30 \%$ du parc de logement de l'aire urbaine sur son territoire de moins de $27,5 \mathrm{~km}^{2}$, se distingue également de sa périphérie urbaine et périurbaine. En 2013, chaque logement "occupe " en moyenne $178 \mathrm{~m}^{2}$ de terrain à Orléans, alors qu'il occupe $553 \mathrm{~m}^{2}$ de terrain en moyenne dans les 21 autres communes du périmètre SCOT qui coïncide ici avec celui de la Communauté d'Agglomération, devenue Métropole en 2017. Le COS résidentiel observé d'Orléans est donc de 0,37 en 2013 contre 0,31 en 1946 dans les limites territoriales de 2013. Inversement, le cos résidentiel observé a diminué dans les communes périphériques de l'agglomération, passant de $0,16 \mathrm{~m}^{2}$ habitable par $\mathrm{m}^{2}$ de terrain en 1946 à 0,12 en 2013. À Orléans comme à Tours, l'étalement urbain concerne principalement les communes périphériques de l'agglomération sur la longue durée d'observation retenue dans cette étude. Au contraire, les deux villes-centres ont cherché à accroître leur offre de logement en se densifiant plus ou moins. Cependant, cette politique a été plus suivie à Tours qu'à Orléans. 


\section{L'impact du nouveau cadre réglementaire à Tours et à Orléans}

On peut faire un premier bilan des effets des changements réglementaires introduits par la loi SRU dans les aires urbaines de Tours et d'Orléans et regardant les caractéristiques des logements neufs achevés dans les 10 dernières années de la période considérée, soit entre 2003 et 2012 (tableau 3).

Tableau 3. Comparaison statistique Orléans/Tours, parc de logement achevé entre 2003 et 2012

\begin{tabular}{|c|c|c|c|c|c|c|}
\hline \multirow[b]{2}{*}{$\begin{array}{l}\text { Logement } \\
\text { selon les } \\
\text { dates de } \\
\text { construction }\end{array}$} & \multicolumn{3}{|c|}{ Aire urbaine de Tours } & \multicolumn{3}{|c|}{ Aire urbaine d'Orléans } \\
\hline & $\begin{array}{l}\text { Ville de } \\
\text { Tours } \\
\text { (délimitation } \\
\text { 2013) }\end{array}$ & $\begin{array}{l}39 \text { communes } \\
\text { périphériques } \\
\text { SCOT de } \\
\text { Tours }\end{array}$ & $\begin{array}{l}\text { Périphérie } \\
\text { de } \\
\text { urbaine l'aire }\end{array}$ & $\begin{array}{l}\text { Ville } \\
\text { d'Orléans } \\
\text { (délimitation } \\
\text { 2013) }\end{array}$ & $\begin{array}{l}21 \text { communes } \\
\text { périphériques } \\
\text { scoT } \\
\text { d'Orléans }\end{array}$ & $\begin{array}{l}\text { Périphérie } \\
\text { de l'aire } \\
\text { urbaine }\end{array}$ \\
\hline $\begin{array}{l}\text { Parc de } \\
\text { logement } \\
\text { construit de } \\
2003 \text { à } 2012 \\
\% \text {, total aire } \\
\text { urbaine } \\
100 \%)\end{array}$ & $\begin{array}{l}5936 \\
(21 \%)\end{array}$ & \begin{tabular}{|l}
14442 \\
$(51 \%)$
\end{tabular} & $\begin{array}{l}8101 \\
(28 \%)\end{array}$ & $\begin{array}{l}4853 \\
(23 \%)\end{array}$ & $\begin{array}{l}8009 \\
(38 \%)\end{array}$ & $\begin{array}{l}8391 \\
(39 \%)\end{array}$ \\
\hline \begin{tabular}{|l|} 
cos \\
résidentiel \\
observé \\
moyen (post \\
2002)
\end{tabular} & 0,77 & 0,16 & 0,09 & 0,56 & 0,21 & 0,10 \\
\hline \begin{tabular}{|l|} 
Superficie \\
moyenne \\
logement \\
ancien (ante \\
1946 )
\end{tabular} & $61 \mathrm{~m}^{2}$ & $93 \mathrm{~m}^{2}$ & $99 \mathrm{~m}^{2}$ & $66 \mathrm{~m}^{2}$ & $92 \mathrm{~m}^{2}$ & $107 \mathrm{~m}^{2}$ \\
\hline $\mid \begin{array}{l}\text { Part des } \\
\text { maisons } \\
\text { (ante 1946) }\end{array}$ & $9 \%$ & $63 \%$ & $88 \%$ & $19 \%$ & $61 \%$ & $93 \%$ \\
\hline
\end{tabular}

En 10 ans, il s'est construit près de 28500 logements neufs dans l'aire urbaine de Tours. On a construit de plus grands logements en dehors de la ville de Tours, et une proportion plus importante de maisons individuelles ont été construites au-delà des limites du périmètre SCOT, qui n'a regroupé que $72 \%$ des constructions neuves. Faute de réserves foncières, la part de la ville de Tours dans la construction a baissé ( $21 \%$ des logements neufs de l'aire urbaine seulement sur les 10 dernières années contre $33 \%$ sur l'ensemble de la période d'étude), et il s'y est construit principalement de petits appartements.

31 Il s'est construit environ 21250 logements neufs dans l'aire urbaine d'Orléans sur la même période. On a construit de plus grands logements en dehors de la ville d'Orléans, et 
une proportion plus importante de maisons individuelles ont été construites au-delà des limites du périmètre SCOT, qui n'a regroupé que $62 \%$ des constructions neuves. La part de la ville-centre dans la construction a baissé ( $23 \%$ des logements neufs de l'aire urbaine seulement sur les 10 dernières années contre $29 \%$ sur l'ensemble de la période d'étude), et il s'y est aussi construit surtout de petits appartements.

Dans les deux aires urbaines, les COS résidentiels observés des constructions récentes sont plus élevés que la moyenne de ce qui a été construit ou programmé avant l'entrée en vigueur de la loi SRU, avec un COS moyen de 0,15 dans l'Orléanais et de 0,14 en Touraine pour les constructions achevées entre 2003 et 2012. Ce gain de densité résidentielle est principalement tiré par les villes-centres, le périurbain reste très peu dense et on observe au contraire une tendance à l'agrandissement des parcelles pour construire des maisons plus grandes, en particulier dans les communes périphériques de l'aire urbaine d'Orléans.

\section{Comparaison des formes de l'extension résidentielle}

En l'absence de forte densité résidentielle, la forme spatiale de l'expansion résidentielle des agglomérations de Tours et d'Orléans revêt donc la forme dominante de l'étalement urbain en tache d'huile.

L'expansion de l'agglomération de Tours permet de repérer une périodisation assez marquée (figure 5).

Figure 5. Agglomération de Tours, carte interactive du parc de logement 1946-2012

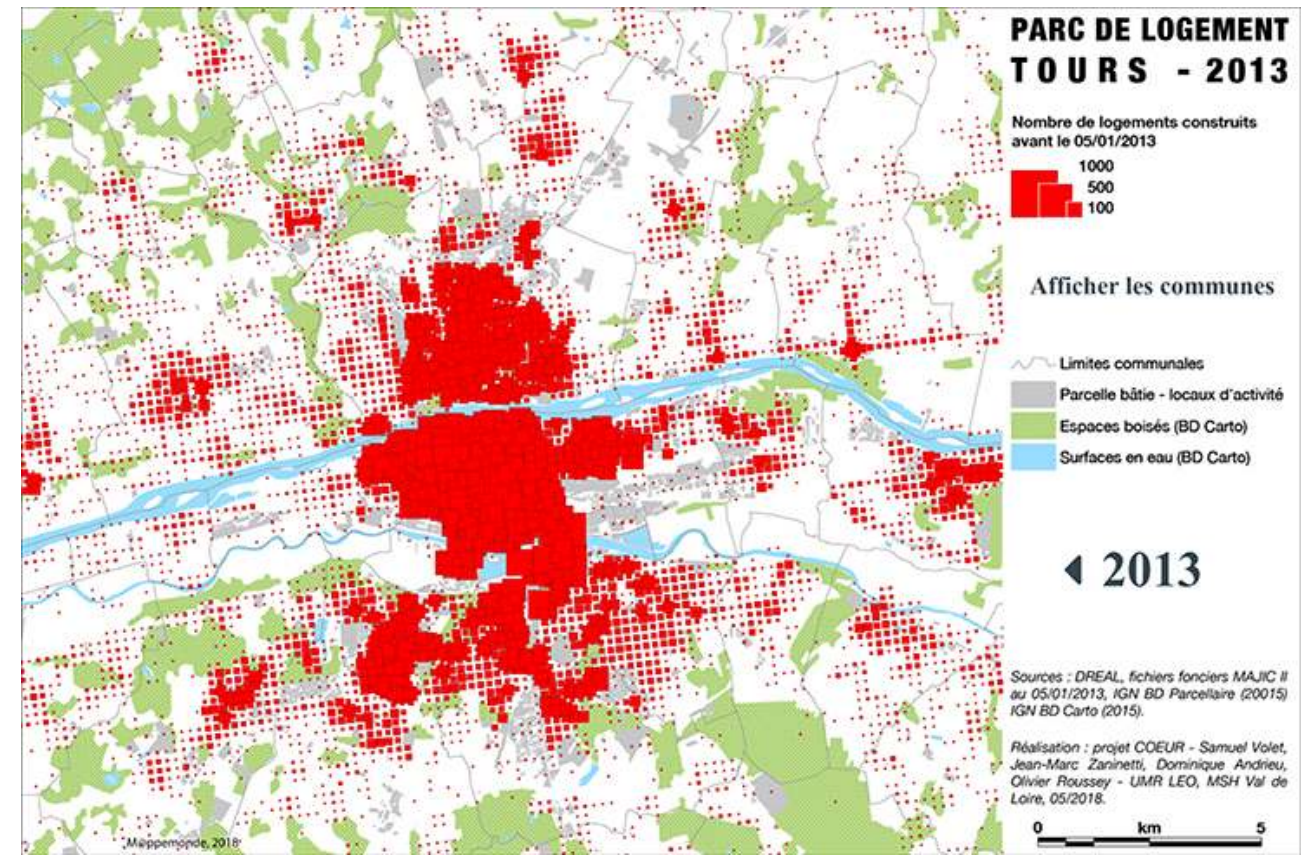

Consulter la carte interactive : http://mappemonde.mgm.fr/geovisu/124/fig5

Le site historique de la ville de Tours se trouve sur la rive gauche de Loire. Au sortir de la Seconde Guerre mondiale, Tours est une ville compacte sise entre Loire et Cher. Des faubourgs peu étendus s'étendent sur la rive nord en face du pont Wilson par lequel la route Paris - Bordeaux franchit le fleuve. La gare ferroviaire est dédoublée à l'est sur la commune de Saint Pierre-des-Corps qui forme la première banlieue de Tours. L'expansion 
spatiale de l'agglomération commence dans les années 1960, avec la construction de grands ensembles périphériques à Tours principalement, dont le territoire communal s'étend surtout sur la rive droite de la Loire, mais aussi à Joué-lès-Tours et à Saint-Pierredes-Corps. Après 1975, la tache d'huile des lotissements pavillonnaires favorise un étalement urbain accéléré, notamment sur les plateaux situés au sud du Cher comme, par exemple, les communes de Saint Avertin et Chambray-lès-Tours qui s'urbanisent en continuité de l'agglomération. Par contre, on observe aussi une extension de tous les villages environnants, qui dessine une deuxième couronne d'expansion périurbaine en "peau de léopard». Cette dernière forme de croissance est prédominante depuis le tournant du siècle, elle est particulièrement visible dans des communes telles que Fondettes ou Montlouis-sur-Loire, par exemple, par l'addition de petites opérations individuelles dans les bourgs existants. Ce mode de développement d'une "deuxième couronne » périurbaine n'est pas nouveau en France (Beaujeu-Garnier, 1980 ; Steinberg, 1991, p. 26.) et la métaphore de la " peau de léopard » était déjà évoquée à cette époque. Mais nos observations de terrain indiquent que la proportion de la construction neuve qui se localise dans ces villages et bourgs de la « deuxième couronne » des aires urbaines a augmenté depuis le tournant du siècle.

Ces tendances à l'étalement urbain sont localement contrariées par de grands projets d'aménagement pilotés par les villes-centres. Dans la ville de Tours, l'aménagement graduel du quartier des Deux Lions, esquissé à la fin du mandat de Jean-Royer, sur la plaine d'inondation de la rive gauche du Cher - une des dernières réserves foncières municipales existant encore à l'époque - prend le parti radical de surélever le terrain pour le rendre constructible (Dumont, 2005), ce qui crée une nouvelle centralité urbaine relativement dense avec la construction de nombreux appartements neufs.

Figure 6. Agglomération d'Orléans, carte interactive du parc de logement 1946-2012

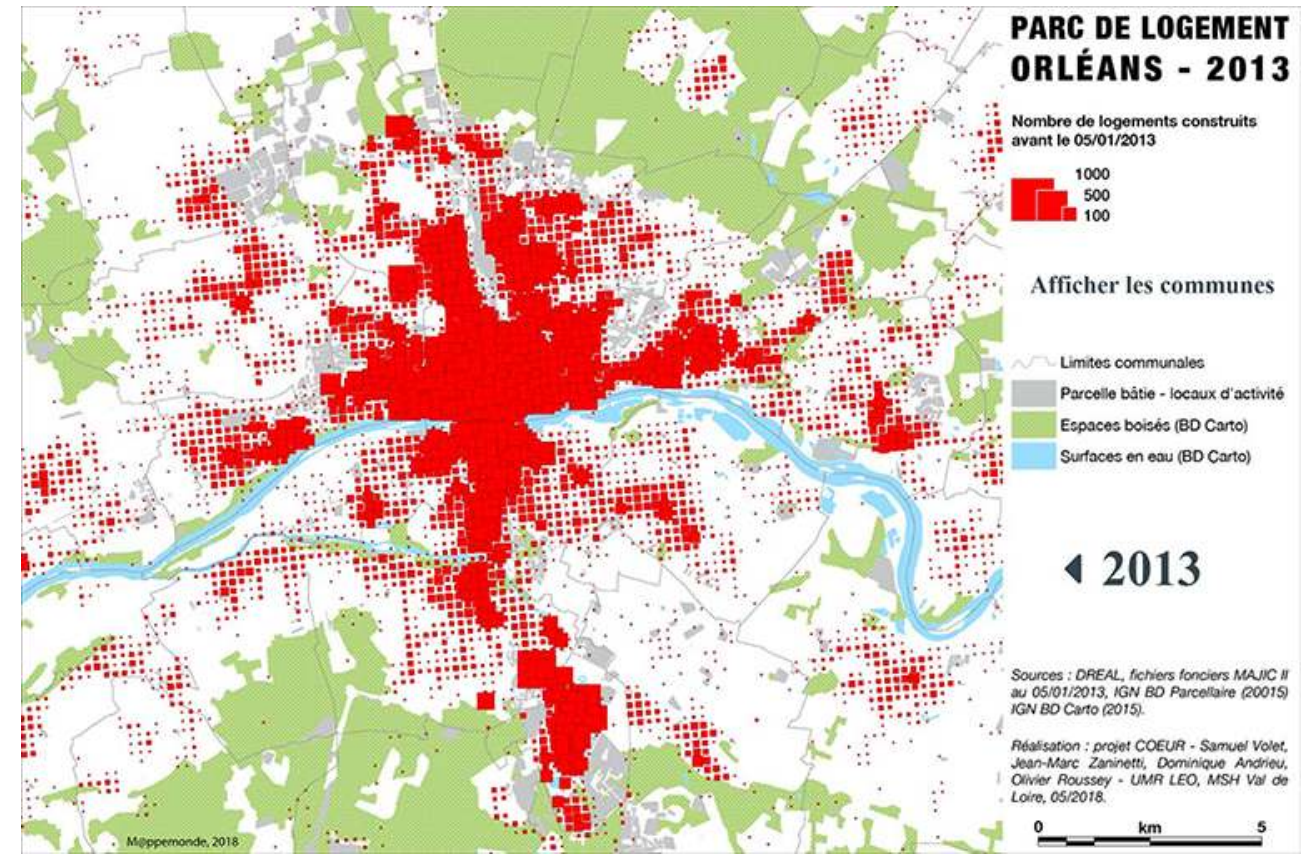

Consulter la carte interactive : http://mappemonde.mgm.fr/geovisu/124/fig6

Pour sa part, le site historique de la ville d'Orléans se trouve sur la rive droite de Loire. Au sortir de la Seconde Guerre mondiale, Orléans est une ville compacte sise au nord de la 
Loire. Des faubourgs peu étendus s'étendent sur la rive sud en face du pont George V par lequel la route Paris - Toulouse franchit le fleuve. La gare ferroviaire est dédoublée au nord sur la commune de Fleury-les-Aubrais qui forme la première banlieue d'Orléans. L'expansion spatiale de l'agglomération commence dans les années 1960, avec la construction de grands ensembles périphériques à Orléans principalement, mais aussi à Saint-Jean-de-la-Ruelle et à Fleury-les-Aubrais. Décidée en 1962, avec le rachat de parcelles aux communes voisines par la ville d'Orléans, l'opération Orléans - La Source est une singularité orléanaise qui se construit dans la période 1962-1982 sur le modèle des villes nouvelles parisiennes. Située sur le plateau non inondable de Sologne au sud du Loiret, cette opération dédouble la centralité orléanaise au sud de la Loire (Grésillon, 1995 ; Mirloup, 2002). Après 1975, la tache d'huile des lotissements pavillonnaires favorise un étalement urbain accéléré jusqu'en 1999 dans toutes les directions. Par contre, on observe aussi une extension de tous les bourgs et villages environnants, dont les plus proches s'agrègent à l'agglomération. Depuis le tournant du siècle, l'empreinte spatiale de l'agglomération est stabilisée, par contre l'urbanisation des dents creuses se poursuit (les urbanistes parlent volontiers "d'opérations cœur d'îlot») et tous les bourgs de l'aire urbaine se développent, bien que l'expansion périurbaine en "peau de léopard » soit de plus faible intensité qu'à Tours.

Selon la classification du CERTU (Der Madirossian, 2010), on peut considérer que le tissu résidentiel est diffus en dessous de 40 logements par hectare, et dense au-dessus de 120 logements par hectare. Selon cette classification, le décompte des logements sur le carroyage de 4 hectares donne les résultats suivants pour les aires urbaines d'Orléans et de Tours au $1^{\text {er }}$ janvier 2013 (tableau 4).

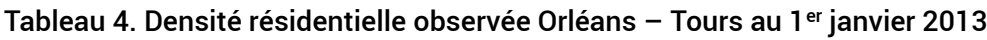

\begin{tabular}{|l|l|l|}
\hline $\begin{array}{l}\text { Densité résidentielle (superficie cadastrale ha) } \\
\text { zone habitée) }\end{array}$ & $\begin{array}{l}\text { Aire urbaine de } \\
\text { Tours }\end{array}$ & $\begin{array}{l}\text { Aire urbaine } \\
\text { d'Orléans }\end{array}$ \\
\hline Pas de logements & 235991 ha & 252079 ha \\
\hline Tissu résidentiel diffus (<40 log./ha) & 65791 ha & 45234 ha \\
\hline Tissu résidentiel intermédiaire (40-120 log./ha) & 948 ha & 756 ha \\
\hline Tissu résidentiel dense (120 log./ha) & 183 ha & 136 ha \\
\hline Superficie totale de l'aire urbaine (ha) & 302912 ha & 298205 ha \\
\hline
\end{tabular}

Source: MAJIC + BD Parcellaire, calculs de l'auteur sous SIG

Les cellules du carroyage possédant au moins 1 logement ne couvrent que $22 \%$ de la superficie cadastrale de l'aire urbaine de Tours. Les quartiers urbains denses ne représentent que $0,27 \%$ de la superficie du bâti résidentiel, tandis que l'urbanisation diffuse représente $98,3 \%$ de sa superficie.

L'espace résidentiel est moins étalé autour d'Orléans, avec seulement $15 \%$ de la superficie cadastrale de l'aire urbaine. L'urbanisation diffuse représente $98,1 \%$ de la superficie du bâti résidentiel, tandis que les quartiers urbains denses ne représentent que $0,3 \%$ de cette 
surface. Toutefois, le noyau urbain d'une densité résidentielle supérieure ou égale à 40 logements par hectare est plus étendu à Tours, ville plus importante, qu'à Orléans.

\section{Discussion}

41 En dépit de gouvernances territoriales assez différentes, Orléans et Tours ont eu des trajectoires d'urbanisation similaires depuis la fin de la Seconde Guerre mondiale. Le phasage identique de leur développement manifeste la prédominance de facteurs économiques comparables et l'influence décisive du cadre réglementaire national. En l'absence de contrainte foncière forte, la densification résidentielle est restée très limitée. On peut même parler d'étalement urbain absolu jusqu'en 1982, date qui marque un point bas historique sur la courbe du cos résidentiel observé dans les deux aires urbaines ( figures 3 et 4). La densification observée depuis, principalement après l'adoption de la loi SRU en 2000, reste encore modeste, et essentiellement limitée au ressort territorial des deux villes-centres, qui ne contrôlent directement qu'une petite fraction du territoire de leur aire urbaine.

$\mathrm{Au}$ terme de cette analyse descriptive sur le long terme, il convient de se demander si les conditions économiques locales sont porteuses pour une stratégie de renouvellement urbain fondé sur la densification résidentielle. Il semble que la densification urbaine ne rencontre pas la demande solvable en dehors d'un centre-ville gentrifié relativement peu étendu (tableau 4). La gouvernance territoriale renforcée dans les périmètres SCOT n'est pas la garantie d'une transition vers plus de densité urbaine si les objectifs des autorités régulatrices ne rencontrent pas des conditions de marché favorables. Pour l'heure, la nouvelle réglementation de l'urbanisme a découragé l'étalement urbain en tache d'huile, mais la demande s'est déplacée vers les petites opérations dans le prolongement des villages et des bourgs existants, en particulier autour de Tours. Le coût marginal de ces opérations d'extension des bourgs et villages est réduit pour les acteurs de la construction, car cette urbanisation éparpillée valorise les infrastructures publiques existantes, et ni les promoteurs, ni les communes n'assument les coûts collectifs de la dépendance automobile, de la congestion routière et - en l'état des technologies actuelles - de la pollution qui en découlent. L'étude des cas de Tours et d'Orléans montre donc que les lois adoptées durant la période 1995-2001 pour renforcer la gouvernance territoriale et réduire l'étalement urbain n'ont pas prévalu sur les conditions du marché dans cette région. Les modalités de production de la ville éparpillée apparues dans les années 1970 se sont adaptées aux nouvelles contraintes, mais persistent tout de même autour des métropoles intermédiaires du Val de Loire.

La demande de maisons individuelles en périphérie dans des lotissements pavillonnaires diffus répond bien à la demande de sociabilité élective des classes populaires salariées qui, après avoir été échaudées par l'expérience des grands ensembles, que beaucoup ont éprouvée avant d'accéder à la propriété d'un pavillon en périphérie, peuplent la «ville émergente » (Dubois-Taine et Chalas, 1997). L'acceptabilité sociale de la densité urbaine n'est pas acquise (Charmes, 2010) et l'étalement diffus participe à une forme de «clubbisation » de l'univers périurbain (Charmes, 2011). Doit-on pour autant considérer ces populations périurbaines comme anti-urbaines? Le malthusianisme foncier de nombreuses communes périurbaines est attesté par la construction de SCOT défensifs en périphérie des grandes agglomérations (Charmes, 2007). C'est le cas à la périphérie d'Orléans, dont l'agglomération relativement restreinte (22 communes) a été assiégée 
après 2001 par trois "pays " périurbains dont les SCOT se définissaient en opposition à l'agglomération centrale. Ces oppositions sont aujourd'hui en passe d'être surmontées, mais notre étude montre que près de $40 \%$ de la construction de logement neuf échappe au SCOT d'Orléans dans son aire urbaine depuis le tournant du siècle.

Il nous semble cependant que ces analyses centrées sur la demande passent à côté de l'essentiel. Comme le rappelle le rapport Decourcelle (2015, p. 16), le choix de l'accession à la propriété en périphérie est d'abord dicté par des considérations financières. Selon l'INSEE, le développement du périurbain a permis aux familles d'accéder à la propriété et à la maison individuelle dans de meilleures conditions économiques, liées à un moindre coût foncier et de construction. Ainsi, entre 2002 et 2006, les ménages accédants ont déboursé en moyenne $136000 €$ pour une maison en zone rurale, $163000 €$ en périurbain contre $200000 €$ dans les grandes agglomérations (Briant, 2010). Dans le contexte où l'acquisition du logement est une dépense fortement encadrée par les banques, un écart moyen de $40000 €$ peut faire toute la différence pour solvabiliser ou non de nombreux ménages candidats à l'accession à la propriété de leur résidence principale. La première conséquence du durcissement récent du droit de la construction semble surtout avoir été de décourager les grandes opérations au profit de l'urbanisation diffuse éparpillée dans les bourgs et villages des "campagnes urbaines» (Castel, 2007). La volonté de densification se heurte à la cherté du foncier urbain et à des surcoûts de construction qui disqualifient les classes moyennes accédantes cherchant à acquérir des appartements en centre-ville (Castel et Jardinier, 2011). Dans ces conditions, on comprend mieux le succès de la construction de logements individuels "purs» dans les bourgs et villages environnants les agglomérations, et la morphologie de l'urbanisation en "peau de léopard » bien visible en particulier autour de Tours dans les décennies récentes.

Les promoteurs immobiliers sont très peu actifs dans la construction de logement neuf en région Centre-Val de Loire. Beaucoup de constructions se font de gré à gré entre l'utilisateur final du logement et un artisan constructeur. Selon les statistiques du ministère du Logement (base de données Sit@del), le logement individuel en maison représente 2 constructions de logement neuf sur 3 autorisées entre 2000 et 2015 en région Centre-Val de Loire. Parmi ces maisons, 3 logements individuels neufs sur 4 autorisés sont des logements individuels "purs ", c'est-à-dire construits à l'unité, hors lotissement. L'autopromotion immobilière (Duncan et Rowe, 1993 ; Halleux, 2011) est favorisée par l'intermédiation des communes qui assurent elles mêmes le portage foncier par la viabilisation des parcelles dans la continuité du bâti existant.

Parvenus à ce stade très préliminaire de notre programme de recherche sur les modalités de la croissance urbaine en région Centre-Val de Loire, nous ne pouvons que nous interroger sur le rôle des marchés dans la poursuite de l'éparpillement urbain observé autour des deux métropoles ligériennes. La poursuite de notre programme requiert de faire des enquêtes sur les prix fonciers, sur les acteurs de la construction et sur les prix de l'immobilier pour avancer dans la compréhension des dynamiques urbaines qui s'y déroulent. Dans l'articulation entre morphologie urbaine et mobilités, il semble que la première, et donc la construction, est décisive, car la mobilité des ménages motorisés s'adapte à la configuration de leur bassin de vie, alors que les choix de localisation résidentielle sont contraints économiquement. Le problème de l'éparpillement urbain actuel est qu'il ne permet pas, dans les conditions présentes, la structuration de ces nouvelles « campagnes urbaines " par un système de mobilité écologique et performant. La «ville éparpillée » génère l'entropie de déplacements en tous sens qui saturent la 
voirie routière existante, inadaptée car dimensionnée pour une circulation rurale et agricole diffuse et non pour des navettes domicile-travail de plus en plus nombreuses. Les pouvoirs publics sont conscients de la nécessité d'aménager les " campagnes urbaines » (Bonnet, 2016). Après avoir fait l'éloge de la ville diffuse, le rapport Decourcelle (2015) propose de "requalifier les campagnes urbaines». Compte tenu de l'ampleur du phénomène dans le Val de Loire, il nous semble que cela devrait également être une priorité de l'action régionale.

\section{BIBLIOGRAPHIE}

BACCAÏNI B., SEMECURBE F. (2009). « La croissance périurbaine depuis 45 ans ». INSEE première, $\mathrm{n}$

$\circ 1240,4 \mathrm{p}$.

BAUER G., ROUX J.-M. (1976). La rurbanisation ou la ville éparpillée. Paris : Édition du Seuil, 189 p. ISBN 9782020043915.

BeAujeu-Garnier J. (1980). Géographie urbaine. Paris : Armand Colin, 360 p.

BERQue A., Bonnin P., GHorra-Gobin C., dir. (2006). La Ville insoutenable. Actes du colloque de Cerisy. Paris : Belin, 365 p., ISBN 978-2-7011-4186-2.

BESSY-PIETRI P. (2000). « Les formes récentes de la croissance urbaine ». Économie et statistique, $\mathrm{n}$ -336, p. 35-52.

BONNET F. (2016). Aménager les territoires ruraux et périurbains. Rapport remis à $\mathrm{M}^{\mathrm{me}}$ la ministre du Logement le 7 janvier 2016. Paris : La Documentation Française, 129 p.

En ligne : http://www.ladocumentationfrancaise.fr/var/storage/rapports-publics/164000021.pdf

BONNEVILLE M. (2004). «Les ambiguïtés du renouvellement urbain en France : Effets d'annonce, continuité ou rupture? ». Les Annales de la recherche urbaine, $n^{\circ}$ 97, Renouvellements urbains, p. 7-16. DOI 10.3406/aru.2004.2571.

BRIANT P. (2010). «L'accession à la propriété dans les années 2000 ». INSEE Première, n 1291, 4 p.

CASTEL J.-C. (2007). « De l'étalement urbain à l'émiettement urbain. Deux tiers des maisons construits en diffus ». Annales de la Recherche urbaine, vol. 102, n 1, p. 88-96.

CASTEL J.-C., JARDINIER L. (2011). « La densité au pluriel ». Études foncières, n 152, p. 13-17.

CHARMES E. (2007). « Les périurbains sont-ils anti-urbains ? Les effets de la fragmentation communale ». Annales de la Recherche urbaine, vol. 102, n 1, p. 7-17.

CHARMes E. (coord.) (2010). « La densification en débat. Effet de mode ou solution durable?». Études foncières, dossier, $\mathrm{n}^{\circ} 145$, p. 20-38.

CHARMES E. (2011). La Ville Émiettée. Essai sur la clubbisation de la vie urbaine. Paris : PUF, coll.« La ville en débats ", $288 \mathrm{p}$.

ChARmes E. (2013). « L'artificialisation est-elle vraiment un problème quantitatif ? ». Études foncières, $\mathrm{n}^{\circ} 162$, p. 23-28. 
Chauveau E., Desire G., Feuillet T., Lowenbruck J.(2012). « Xynthia, du drame subi à une anticipation des évènements rares : outils et perspectives ». Paris : CEREMA, Congrès SHF : «Évènements extrêmes fluviaux et maritimes », Paris, 1-2 février 2012.

En ligne : http://www.side.developpement-durable.gouv.fr/EXPLOITATION/DRPDLO/doc/IFD/ IFD_REFDOC_0512351/xynthia-du-drame-subi-a-une-anticipation-des-evenements-rares-outilset-perspectives.

Сомву J. (2004). «Quelques idées simples sur les politiques foncières locales ». Études foncières, $\mathrm{n}$ - 110 , p. 7-8.

Decourcelle J.-P., NARring P., Peyrat J. (2015). Requalifier les campagnes urbaines de France. Une stratégie pour la gestion des franges et des territoires périurbains. CGEDD, Rapport $\mathrm{n}^{\circ}$ 009794-01, $124 \mathrm{p}$.

Der MAdirossian L. (2010). La densité urbaine. Plaquette pédagogique. CERTU, 12 p.

DESJARDINS X. (2010). « Que retenir de La Courbe de Newman et Kenworthy? ». Études Foncières, n - 145, p. 27-29.

DJellouli Y., Emelianoff C., BennasR A., CheVAlieR J., coord. (2010). L'étalement urbain. Un processus incontrôlable? Rennes : PUR, coll. « Espace et Territoires », 260 p. ISBN 978-2-7535-1049-4.

DREAL FRANCHE COMTÉ (2013). Outil dynamique d'urbanisation : bâti et année de construction. En ligne : http://cartelie.application.developpement-durable.gouv.fr/cartelie/voir.do? carte=Cartelie_Dynamique_urbaine_v2\&service=DREAL_Fr_Comte.

DREVELLE M. (2012). «Structure des navettes domicile-travail et polarités secondaires autour de Montpellier ». M@ppemonde, $\mathrm{n}^{\circ}$ 107, 2012-3.

En ligne : http://mappemonde.mgm.fr/num35/articles/art12304.html.

Dubois-TAine G., Chalas Y., dir. (1997). La ville émergente. La Tour d'Aigues : DATAR/ Éditions de l'Aube, 286 p. ISBN 2-87678-329-0

Dumont M. (2005). « Le développement urbain dans les villes intermédiaires : pratiques métropolitaines ou nouveau modèle spécifique ? Le cas d'Orléans et Tours ». Annales de géographie, $\mathrm{n}^{\circ}$ 642, 2005-2, p. 141-162. DOI 10.3917/ag.642.0141.

DunCAN S.S., Rowe A. (1993). "Self-provided housing: The first World's Hidden Housing Arm". Urban Studies, vol. 30, $\mathrm{n}^{\circ}$ 8, p. 1331-1354.

DuPUY G. (1999). La dépendance automobile. Symptômes, analyses, diagnostic, traitements. Paris : Anthropos, coll. « Villes », 160 p.

DUVERNOY I. (2002). « Espace agricole périurbain et politiques communales d'aménagement: L'exemple de l'agglomération albigeoise ». Cybergeo : European Journal of Geography, Aménagement, Urbanisme, document 208, mis en ligne le 5 mars 2002, consulté le 20 mars 2018. DOI 10.4000/ cybergeo.1965.

En ligne : http://journals.openedition.org/cybergeo/1965.

FABLET G. (2013). « La croissance immobilière des stations de sports d'hiver en Tarentaise. Entre vulnérabilités conjoncturelles et dérèglements structurels ». Revue de géographie alpine, $\mathrm{n}^{\circ}$ 101-3, mis en ligne le 24 mars 2014, consulté le 19 mai 2017. DOI 10.4000/rga.2188. En ligne : http:// rga.revues.org/2188.

FLOCH J-M., LÉVY D. (2011). « Le nouveau zonage en aires urbaines de 2010. Poursuite de la périurbanisation et croissance des grandes aires urbaines ». INSEE Première, $n^{\circ} 1375.4$ p.

FOUCHIER V. (1998). Les densités urbaines et le développement durable : le cas de l'Ile de France et des villes nouvelles. Paris : Secrétariat général du groupe central des villes nouvelles, $211 \mathrm{p}$. 
GiLLi F. (2006). « Vingt ans de dépenses publiques de logement ». INSEE, coll « Données sociales : La société française », p. 485-493.

GozE M. (2002). « La stratégie territoriale de la loi SRU ». Revue d'économie régionale et urbaine, $\mathrm{n}$ ०2002-5, p. 761-776.

GRÉSILLON M. (1995). Orléans. Paris : Anthropos, 160 p. ISBN 9782717828399.

GuignaRd M., MARIE J.-B., coord. (2017). « Densification en controverses ». Premier Plan. Le journal d'information du PUCA Plan Urbanisme Construction Architecture, $\mathrm{n}^{\circ}$ 36, dossier, $16 \mathrm{p}$.

HALLEUX J.-M. (2011). Structuration spatiale des marchés fonciers et étalement urbain. Sarrebruck : Éditions universitaires européennes, 444 p. ISBN 978-3-8417-8373-8

IETI CONSULTANTS (2008). Les fichiers fonciers standards délivrés par la DGI appelés communément fichiers MAJIC II. Volume1 : Guide méthodologique pour leur utilisation. CERTU, « Les rapports d'étude ", $64 \mathrm{p}$.

Indovina F. (1990). "La Città diffusa". In Indovina F., MATASSONi F., SAVino M., SERnini M., ToRres M., VetToRetTo L., La città diffusa, Venise: Daest-IUAV, quaderno nº 1, p. 21-43.

JULIEN P. (2000). « Mesurer un univers urbain en expansion ». Économie et statistique, $\mathrm{n}^{\circ} 336$, p. 3-33.

JULIEN P. (2007). Analyse critique de la pertinence de l'aire urbaine pour étudier l'étalement urbain. Quelques éléments de prospective. Espaces sous influence urbaine. CERTU, «Les rapports d'étude » $42 \mathrm{p}$.

LAMBert A. (2013). « La gauche et le périurbain ». Politix, n 101, 2013-1, p. 105-131. DOI 10.3917/ pox.101.0105

LANG R.E. (2002). Edgeless Cities. Exploring the elusive metropolis. Washington DC: Brookings Institution Press, 154 p. ISBN 9780815706113

Le Berre I., Maulpoix A., Thériault M., Gourmelon F. (2016). « Une base de données pour modéliser l'urbanisation résidentielle en zone côtière ». In EMSELLEM K., MORENO SIERRA D., VOIRON -CANICIO C., JossELIN D. Actes de la conférence SAGEO 2016. Spatial Analysis and Geomatics, p. 215-228. En ligne

LE JEANNIC T. (1997). «Trente ans de périurbanisation : extension et dilution des villes ». Économie et statistique, $\mathrm{n}^{\circ} 307$, p. 21-41. DOI 10.3406/estat.1997.2578

LEVESQUe R., LIORIT D., PATHIER G. (2011). « Les marchés fonciers ruraux régionaux entre dynamiques des exploitations agricoles et logiques urbaines ». Économie et statistique, $\mathrm{n}^{\circ}$ 444-445, p. 75-98. DOI 10.3406/estat.2011.9644

LÉVY J. (2013). Réinventer la France : trente cartes pour une nouvelle géographie. Paris : Fayard, 245 p. ISBN 978-2213671970.

LusSAUlT M. (1993). Tours : images de la ville et politique urbaine. Tours : Maison des sciences de la ville/Université François Rabelais coll. «Sciences de la ville », 412 p. ISBN 2-86906-054-8.

Mayoux J., dir. (1979). Demain l'espace. L'habitat individuel périurbain. Rapport de la mission d'étude sur l'habitat individuel péri-urbain. Paris : La Documentation Française, coll. «Études prioritaires interministérielles ", $143 \mathrm{p}$.

MiRLouP J. (2002). « Orléans. Éléments d'un système métropolitain ». Mappemonde nº 68, 2002-4, $9 \mathrm{p}$.

En ligne : http://www.mgm.fr/PUB/Mappemonde/M402/Mirloup.pdf. 
NESSI H., LE NÉCHET F., TERRAL L. (2016). «Changement de regard sur le périurbain, quelles marges de manœuvre en matière de durabilité ? ». Géographie, économie, société, vol. 18, p. 15-33.

Newman P., KenWorthy J. (1999). Sustainability and Cities. Overcoming Automobile Dependence. Washington, DC : Island Press, 442 p. ISBN 9781559636605.

Polombo N., BeauVAis J.-M. (2014). « Le tramway de Tours : 1913-2013, un siècle entre 2 tramways. Utilisation simple de MAJIC II pour la représentation de la tache urbaine ». SIG 2014. Conférence francophone ESRI, session « Aménagement et urbanisme », oct. 2014, Versailles, France, 9 p. En ligne : https://halshs.archives-ouvertes.fr/halshs-01060337/document.

PoUYANNE G. (2004). «Des avantages comparatifs de la ville compacte à l'interaction mobilité forme urbaine. Méthodologie et premiers résultats ». Cahiers scientifiques du Transport, $n^{\circ} 45 / 2004$. p. 49-82.

PUCA (2014) « Le périurbain face à ses clichés ». Le quatre pages, $\mathrm{n}^{\circ}$ 17, 4 p.

RENARD V. (2003). « Les politiques foncières de la mixité ». Les Annales de la recherche urbaine, vol. $93, \mathrm{n}^{\circ} 1$, p. 168-173.

Roux J.M. (2006). Des villes sans politique. Étalement urbain, crise sociale et projets. Nantes : Gulf Stream éditeur, 153 p. ISBN 978-2909421513.

SAINTENY G. (2008). « L'étalement urbain ». Annales des Mines. Responsabilité et environnement, $\mathrm{n}^{\circ} 49$, 2008-1, p. 7-15.

STEINBERG J. (1991). « Définition et essai de délimitation. La situation des zones périurbaines ». In DÉZeRt B., MetTon A., STEInBerg J., La périurbanisation en France. Paris : SEDES, p. 21-36.

TAFFIN C. (1987). «L'accession à tout prix ». Économie et statistique, n² 202, p. 5-15.

Touati A., Crozy J. (2015). La densification résidentielle au service du renouvellement urbain : filières, stratégies et outils. Paris : La Documentation française, 150p.

WIEL M. (1999). La transition urbaine ou le passage de la ville pédestre à la ville motorisée. Sprimont : Éditions Pierre Mardaga, 149 p. ISBN 9782870097038.

WIEL M. (2002). Ville et automobile. Paris : Éditions Descartes et C ${ }^{\mathrm{ie}}$, 144 p. ISBN 978-2-87623-033-2

ZANINETTI J.-M., MARET I., dir. (2008). Étalement urbain et ville fragmentée à travers le monde. Orléans : PUO, $256 \mathrm{p}$

ZANINETTI J.-M, (2017). « Les déplacements domicile-travail structurent-ils encore les territoires?»M@ppemonde, $\mathrm{n}^{\circ}$ 122, 18 p. En ligne : http://mappemonde.mgm.fr/122as2.

\section{NOTES}

1. http://www.geoinformations.developpement-durable.gouv.fr/fichiers-fonciers-r549.html.

2. La loi du 28 février 2017 relative au statut de Paris et à l'aménagement métropolitain a élargi la possibilité de transformation en métropole à tous les EPCI de plus de 400000 habitants, tous les EPCI qui sont centres d'une zone d'emplois de plus de 400000 habitants et les EPCI de plus de 250000 habitants ou comprenant dans leur périmètre, au 31 décembre 2015, le chef-lieu de région, centres d'une zone d'emplois de plus de 500000 habitants. Ceci a donné aux intercommunalités d'Orléans et de Tours la possibilité d'accéder au statut convoité de «métropole ». C'est chose faite pour Tours depuis le 22 mars 2017, et pour Orléans depuis le $1^{\text {er }}$ mai 2017. 


\section{RÉSUMÉS}

Les métropoles ligériennes de Tours et Orléans sont deux exemples représentatifs de l'éparpillement résidentiel des villes survenu en France après la Seconde Guerre mondiale. Tandis que le législateur renforce l'arsenal réglementaire en faveur de la densification résidentielle depuis le tournant du siècle, les aires urbaines de Tours et d'Orléans ne manifestent qu'un début timide de densification avant 2013. En s'appuyant sur les fichiers fonciers MAJIC, la comparaison des deux aires urbaines manifeste plus de similitudes que de différences en dépit d'acteurs politiques et de gouvernances territoriales très différentes. La chronologie de la construction de logement autour des deux villes ligériennes est rythmée par les grandes inflexions des politiques nationales de logement. Tandis que les villes-centres - surtout Tours font un effort manifeste de densification urbaine, les autres communes privilégient encore l'éparpillement périphérique des maisons individuelles dans les campagnes urbaines environnantes, dessinant une croissance "en peau de léopard» autour des villages. Avec un foncier disponible abondant et pas cher ainsi que des coûts de construction réduits, la construction diffuse de pavillons individuels semble bénéficier d'un avantage économique difficile à concurrencer pour les promoteurs du renouvellement urbain qui tentent de commercialiser des appartements dans de nouveaux quartiers urbains denses.

The Loire Valley cities of Tours and Orleans are good examples of France's urban housing sprawl after WW2. Since the turn of the century, French authorities have tightened urban development regulations to support residential densification. Cities like Tours and Orléans barely showed a change in development before 2013. Using MAJIC's real estate data, a comparison of the two metropolitan areas displays more resemblance than differences despite very different political arrangements and different metropolitan governance. The timeline of housing development reflects nationwide housing policies. While central cities - notably Tours - actively promote housing densification, other communities still rely on scattered greenfield development of single family homes in the outlying "urban countryside", resulting in a spotted, "leopard-skin" pattern of sprawling villages. With abundant and affordable land and reduced construction costs, the development of individual homes has a competitive edge that is hard to overcome for promoters of urban "renewal" trying to sell apartments in new, densely populated urban neighbourhoods.

Las metrópolis ligerienses de Tours (Turs) y de Orleans son claros ejemplos de la dispersión residencial que se produce en Francia tras la Segunda Guerra Mundial. Mientras que la reglamentación urbana y la legislación favorecen la compacidad y densidad residencial, estas sólo se producen con el cambio de siglo, y sólo muy tímidamente a partir del 2013. Mediante la información catastral de MAJIC se han comparado ambas metrópolis, que presentan más similitudes que divergencia, a pesar las grandes diferencias de sus actores sociales y modelos de gobernanza. La urbanización residencial alrededor estas dos ciudades ligerienses han seguido los tempos e inflexiones de la política nacional de la vivienda. Mientras que sus ciudades centrales especialmente Tours- hicieron un verdadero esfuerzo por aumentar su densidad urbana, los municipios de sus periferias priorizaron la dispersión de las vivienda individuales en sus entornos rurales, fomentando alrededor de los pueblos una leopardización de crecimiento difuso. La disponibilidad de tierras con precios bajos y costes de contrucción reducidos favorecieron la 
vivienda individual. Esta presenta unas ventajas comparativas para los promotores frente a la renovación urbana donde se oferta apartamentos en nuevos barrios con densidades más altas.

INDEX

Mots-clés : densité urbaine, étalement urbain, gouvernance territoriale, périurbanisation, renouvellement urbain

Keywords : urban spread, local governance, urban density, suburbanization, urban renewal

Palabras claves : expansión urbana, gobernanza territorial, densidad urbana, periurbanización, renovación urbana

\section{AUTEURS}

\section{JEAN-MARC ZANINETTI}

Professeur des universités, Université d'Orléans/CNRS, UMR 7322 LEO

DOMINIQUE ANDRIEU

Maison des Sciences Humaines Val-de-Loire 